\title{
Élites urbaines et élites impériales sous Zénon (474-491) et
} Anastase (474-518)

Vincent Puech

\section{Citer ce document / Cite this document :}

Puech Vincent. Élites urbaines et élites impériales sous Zénon (474-491) et Anastase (474-518) . In: Topoi, volume 15/1, 2007. pp. 379-396;

doi : https://doi.org/10.3406/topoi.2007.2243

https://www.persee.fr/doc/topoi_1161-9473_2007_num_15_1_2243

Fichier pdf généré le 27/10/2018 


\section{ÉLITES IMPÉRIALES ET ÉLITES URBAINES SOUS ZÉNON (474-491) ET ANASTASE (491-518)}

Dans sa synthèse Die Spätantike A. Demandt inclut les deux règnes de Zénon et Anastase dans un chapitre chronologique intitulé « Die Krise im Ostreich (450518) ${ }^{1}$. Davantage qu'aux menaces extérieures qui minent l'Occident romain contemporain, la crise de l'Empire d'Orient renvoie alors à des phénomènes de dissensions intérieures. Il semble que les débats post-chalcédoniens et la puissance des Isauriens se conjuguent pour ébranler le solide édifice bâti par Constantin et Théodose. Si ces deux facteurs religieux et militaire sont maintenant de mieux en mieux connus, il reste à reconsidérer leur contexte proprement politique. En la matière, la méthode prosopographique est d'un secours précieux car elle offre une vue d'ensemble des protagonistes de ces deux règnes, tant des fidèles des empereurs que de leurs opposants. Nous sommes donc parti de la Prosopography of the Later Roman Empire ${ }^{2}$, qui a été finalement assez peu utilisée dans son usage le plus direct, l'histoire du personnel politique. Comme l'impose la conception de cet instrument de travail, nous n'avons pas poussé l'enquête aussi loin qu'il aurait fallu au sujet des personnages ecclésiastiques. En outre, nous ne prétendons pas, dans le cadre de cette brève approche, réinterpréter véritablement ces deux règnes, mais seulement poser quelques jalons à la lumière de la prosopographie ${ }^{3}$.

Un réexamen de l'histoire politique de ces deux règnes au moyen d'une analyse des élites impériales gagne à envisager la notion d'élites urbaines. Cette

1. Demandt 1989.

2. Les deux règnes étudiés relèvent de son second volume : PLRE. Les notices des personnages seront citées précédées de l'abréviation PLRE, et, le cas échéant, suivant la convention typographique attribuée par l'ouvrage à leur grade sénatorial : illustris, SPECTABILIS, CLARISSIMUS. On se reportera par ailleurs à l'ouvrage pour la chronologie détaillée de leurs carrières.

3. L'étude des usurpations en particulier met en cause des facteurs structurels qui débordent largement notre propos : poids de la fiscalité, organisation militaire, implantation de courants théologiques par exemple. 
expression au pluriel recouvre une diversité à la fois géographique et sociologique. Sur le plan spatial, les élites constantinopolitaines ne peuvent être coupées de leur origine, de leur implantation ou de leur champ d'action liés aux villes des provinces. Cette pluralité géographique revêt également un aspect sociologique : la variété des sociétés locales influe sur le visage des élites impériales. Le questionnement retenu implique donc des relations dialectiques entre la capitale, la ville du pouvoir impérial, et les villes des provinces, viviers d'élites pour Constantinople. Les protagonistes des règnes de Zénon et Anastase peuvent être examinés grâce à un triple prisme : leurs liens familiaux, leur enracinement géographique et leurs options religieuses ${ }^{4}$. Pour chacun de ces trois facteurs de regroupement des élites, il faudra tenter de déterminer ce qu'ils doivent à la capitale et aux villes des provinces.

\section{Le règne de Zénon (474-491)}

\section{L'assise dynastique des usurpations}

Les trois usurpations du pouvoir intervenues pendant le règne de Zénon mettent en cause des protagonistes et des motivations en partie différents. Mais elles s'insèrent toutes trois dans la parentèle de l'empereur Léon $1^{\text {er }}$ : il apparaît ainsi qu'un vaste réseau familial fut porté pendant la quasi totalité du règne de Zénon à contester son pouvoir, au nom des droits d'une dynastie ${ }^{5}$. Ainsi, Basiliscus ${ }^{6}$ fut proclamé évidemment en tant que frère de l'impératrice Vérine ${ }^{7}$, épouse de Léon $1^{\text {er }}$, qui plus est par les soins de cette dernière. Ils reçurent dans un premier temps l'appui de leur neveu Armatus ${ }^{8}$, qui finit cependant par les trahir. Il est vrai

4. Elton 2000, p. 404, relie ainsi ces trois types de données à la définition de factions politiques : « Political survival and success in late antiquity (wether in civil, military, ecclesiastical or court politics) depended on one's allies. Alliances of individuals, here described as factions, could be based on many factors, including regional origin, religion, common experience, patronage and marriage. Such alliances were not permanent, but were flexible and mutating relationships in which no aspect was automatically dominant » (c'est nous qui soulignons).

5. DAGRON 1996, p. 47, évoque le « manque de perspective dynastique » des empereurs régnants, en raison des nombreux décès d'héritiers en bas âge dans la descendance de Léon $1^{\text {er }}$.

6. PLRE : Fl. Basiliscus 2. Basiliscus fit en même temps proclamer César son fils (PLRE : Marcus 4).

7. PLRE : Aelia Verina. Vérine intercéda déjà sous le règne de Léon $1^{\text {er }}$ en faveur de son frère Basiliscus à la suite de sa défaite contre les Vandales.

8. PLRE : Armatus. Le personnage reçut pour prix de son soutien le poste de maître des milices praesentalis, qu'il conserva après l'échec de la révolte. Il partagea le 
que son rôle dans le réseau familial était déjà trouble puisqu'il passait pour l'amant de Zenonis ${ }^{9}$, l'épouse de Basiliscus ${ }^{10}$ ! Basiliscus et Vérine furent aussi soutenus par leur beau-frère, un sénateur nommé Zuzus ${ }^{11}$. Enfin, la révolte fut appuyée par Marcianus ${ }^{12}$ et par Illus ${ }^{13}$ qui utilisèrent eux aussi directement, quelques années plus tard, la légitimité de Léon $1^{\mathrm{er}}$.

Le relais fut pris par Marcianus, qui comptait déjà au nombre des partisans de Basiliscus. Ses prétentions impériales lui furent suggérées par son mariage avec Leontia ${ }^{14}$, fille porphyrogénète de Léon $1^{\mathrm{er}}$, alors que l'impératrice Ariane ${ }^{15}$, l'épouse de Zénon, n'était, elle, pas née dans la pourpre. Leontia appuya nettement la révolte de son époux, au point d'être emprisonnée comme lui. De son côté, Marcianus fut soutenu par son propre frère Anthemius ${ }^{16}$. En revanche, lors de cette révolte de Marcianus, Illus demeura dans le camp de Zénon, dont il fut même le principal appui militaire.

Enfin, la révolte de Leontius fut, elle aussi, redevable à la légitimité de l'impératrice Vérine, qui proclama là encore le rebelle. C'est dans ce cas le réseau familial d'Illus qui forma le socle de la rébellion. Le beau-frère d'Illus, Matronianus ${ }^{17}$, fut chargé par lui de la protection de Vérine en 478, puis se joignit aux révoltés en 484 . En outre, Illus fut appuyé par son propre frère Trocundes ${ }^{18}$.

consulat avec Basiliscus.

9. PLRE : Aelia Zenonis.

10. LESZKA 2000 invite à douter de cette tradition rapportée par la Suda qui l'insère dans un portrait systématiquement critique du personnage. La trahison d'Armatus fut évidemment liée à une négociation avec Zénon, qui proposa que le fils d'Armatus, nommé Basiliscus comme le rebelle, devienne César et héritier présomptif du trône.

11. PLRE : ZUZUS.

12. PLRE : Fl. Marcianus 17.

13. PLRE : Illus 1. Illus lui-même serait, selon les données de Malalas, l'oncle maternel de Zénon, et donc apparenté à la famille d'Ariane et de Léon $1^{\text {er }}$ : Demandt 1989, p. 188, n. 29, contra PLRE. Le doute demeure car Malalas est la seule source évoquant ce lien familial : ELTON 2000, p. 400, n. 28.

14. PLRE : Leontia 1.

15. PLRE : Aelia Ariadne.

16. PLRE : Procopius Anthemius 9.

17. PLRE : Matronianus 1.

18. PLRE : Fl. Appalius Illus Trocundes. Le personnage était déjà aux côtés d'Illus lors de l'usurpation de Basiliscus. 
Enfin, il fit libérer de sa prison isaurienne Marcianus, preuve qu'il espérait sans doute bénéficier de son concours ; le personnage se montra visiblement encombrant puisqu'il fut relégué en Italie par les rebelles.

\section{Les facteurs théologiques et géographiques des usurpations}

Les trois révoltes du règne de Zénon présentent par ailleurs des visages bien différents. La rébellion de Basiliscus se caractérisa par un ferme appui sur les monophysites. Du point de vue théologique, on sait que l'Encyclique de Basiliscus fut directement inspirée par l'ancien patriarche d'Alexandrie, Timothée Elure, qui retrouva son poste ${ }^{19}$. Or il apparaît bien que Basiliscus s'est tout particulièrement appuyé sur les proches de Timothée Elure. Ainsi, avant même sa révolte, Basiliscus était soutenu dans ses ambitions par Aspar ${ }^{20}$ : ce personnage, lui-même arien, était par ailleurs le protecteur notoire d'évêques monophysites, au nombre desquels se trouvait bien sûr Timothée ${ }^{21}$, mais aussi Amphilochius de Sidè ${ }^{22}$. De manière plus directe encore, cette fois-ci au moment même de la rébellion, Theoctistus ${ }^{23}$, frère du moine alexandrin Theopompus, devint le maître des offices de Basiliscus ; or ce personnage soutenait les moines monophysites égyptiens proches de Timothée Elure, dont il protégea la réinstallation au patriarcat d'Alexandrie ${ }^{24}$. Enfin, l'Encyclique elle-même fut rédigée par le sophiste Paulus ${ }^{25}$, qui devint moine la

19. Maraval 1998, p. 115. Redies 1997 montre cependant que lors de la première année de règne de Zénon, le patriarche Acace et l'empereur lui-même inclinaient vers les monophysites : de ce point de vue, la révolte de Basiliscus n'impliquait pas nécessairement une opposition frontale au pouvoir en place; elle a même pu être paradoxalement favorisée par l'orientation théologique de ce dernier.

20. PLRE: Fl. Ardabur Aspar. Theophanes, p. 179-180: Aspar promit en 461 à Basiliscus de le porter au pouvoir s'il accordait ses faveurs aux soldats goths. Procope, p. 48, indique à propos de l'expédition de 468 : «On dit, à la vérité, qu'Aspar craignit à ce moment-là qu'une défaite des Vandales ne permît à Léon de renforcer son autorité et de lui donner une assise des plus solides, et qu'en conséquence il adressa mille recommandations à Basiliskos ».

21. Théophane, p. 173 : Aspar s'est opposé en 452 à la déposition de Timothée ordonnée par Léon $1^{\text {er. }}$.

22. Zacharias Rhetor, $H E$ IV, 7, p. 124 : pour Aspar, Amphilochius doit être préservé du même danger que celui qui frappe Timothée.

23. PLRE : Theoctistus 3.

24. Zacharias Rhetor, $H E \mathrm{~V}, 1$, p. 145. De plus, la foule chalcédonienne de Constantinople demanda son exécution : Vie de Daniel le Stylite, 83.

25. PLRE : Paulus 22. 
même année et, plus tard, higoumène à Alexandrie, ce qui montre bien l'importance du groupe égyptien qui entourait Timothée Elure et appuyait Basiliscus ${ }^{26}$.

La révolte de Leontius ${ }^{27}$ fut marquée, elle, par un enracinement très fort en Isaurie, dans une moindre mesure en Syrie du nord et, enfin, de manière plus inattendue, en Égypte. Le groupe isaurien est évidemment d'abord composé des deux principaux rebelles eux-mêmes, Leontius et Illus, tous deux nés dans cette province. Illus possédait dans la Cilicie proche, à Akkala, un domaine foncier, dont D. Feissel a récemment édité un témoignage épigraphique ${ }^{28}$. Le frère et partisan d'Illus, Aspalius ${ }^{29}$, a été enterré en Isaurie, preuve de l'attachement durable de la famille à cette région. Quant à Marsus ${ }^{30}$, autre personnage d'importance puisqu'il présenta le lettré païen Pamprepius ${ }^{31}$ à Illus, il était également isaurien de naissance.

Le deuxième groupe est centré sur Antioche, dont est originaire la famille par alliance d'Illus. Son épouse Asteria ${ }^{32}$, née à Antioche, a fini ses jours dans un monastère de Tarse, en compagnie de sa fille Thecla, qui porte un nom hautement évocateur d'un culte local. Le frère d'Asteria, Matronianus, antiochéen également bien sûr, appuya, comme on sait, les deux rebelles ${ }^{33}$. Le propre frère

26. GRILLMEIER 1990, p. 342, montre cependant que des dissensions survinrent entre monophysites : les moines eutychiens de Constantinople, hostiles à l'Encyclique, intriguèrent auprès de Zenonis, l'épouse de Basiliscus. Mais cela ne fit manifestement que renforcer le soutien à l'usurpateur de Timothée Elure, qui regagna Alexandrie face à cette fronde monastique. Pour Blaudeau 1996, le patriarche d'Alexandrie fut bien le promoteur d'une Église impériale et universelle alternative où le premier rang revenait à son siège épiscopal.

27. PLRE : Leontius 17.

28. FeISSEL 1999.

29. PLRE : Aspalius : sans doute à corriger en Appalius, sur le modèle de son frère Fl. Appalius Illus Trocundes.

30. PLRE : Marsus 2.

31. PLRE : Pamprepius. Trombley 1993-1994, II, p. 21, signale que les décurions païens d'Aphrodisias apportèrent leur appui à la révolte d'Illus, d'après Zacharias Rhetor, Vita Severi, p. 40 : la source cite deux frères, le scholasticos Démocharès et le sophiste Proclos. On voit les multiples ramifications du mouvement en Asie Mineure.

32. PLRE : Asteria.

33. Selon Malalas, fragments : 35, c'est précisément lors de leur occupation d'Antioche en 484 que Matronianus accompagna les rebelles. 
d'Illus, Trocundes, se trouvait certainement en Syrie du nord entre 482 et $484{ }^{34}$. Enfin, Iustinianus ${ }^{35}$, nommé comte des largesses sacrées par Leontius, appuya précisément les deux révoltés en 484 quand ils occupèrent Antioche ${ }^{36}$. D'autre part, la rébellion trouva le concours du patriarche d'Antioche Calandion ${ }^{37}$.

Le troisième point d'appui de Leontius et Illus fut Alexandrie. La révolte rencontra la bienveillance des deux principales autorités de la ville puisqu'il s'agissait du préfet augustal Theognostus ${ }^{38}$ et du patriarche Jean Talaia ${ }^{39}$. Or il est intéressant de noter que le premier a précisément joué un rôle décisif dans l'accès du second à l'épiscopat ${ }^{40}$. De plus, Jean Talaia comme Calandion d'Antioche étaient des chalcédoniens stricts, preuve que les rebelles surent s'appuyer sur une résistance à l'Hénotique promu par l'empereur ${ }^{41}$. Réciproquement, les rebelles avaient, dès avant la révolte, déjà soutenu les chalcédoniens. Le frère d'Illus, Trocundes, dès le début du règne, en 476/477, contribua militairement à la

34. LEMERLE 1963 : inscription sur un bronze, peut-être un pied de candélabre, provenant certainement d'el-Bara, et comportant les titres de l'apogée de sa carrière : V(ir) . INL(lustris) COM(es) . ET . MAG(ister) . VTR(ius) . Q(ue). MIL(itiae) . PATR(icius) . ET . CONS(ul) . O/RD(inarius) .

\section{PLRE : Iustinianus 5.}

36. Malalas, fragment 35 : l'auteur lui-même est issu du milieu antiochéen qu'il connaît bien.

37. Evagrius, III, 16. A.-J. Festugière propose la traduction suivante : «[Calandion] passait pour avoir donné de l'aide à Illus, Léonce et Pamprepius dans leur lutte contre le gouvernement tyrannique de Zénon »; si un doute est possible sur la participation de l'évêque à la révolte, il n'est en tout cas pas lié à une calomnie de la part de l'historien, qui dénigre l'empereur en place. Un signe qui ne trompe guère est le fait que Calandion ait rayé des diptyques le nom de l'empereur, ce qui constitue le symbole même de la contestation du pouvoir.

38. PLRE : Theognostus 2. Zacharias Rhetor, $H E \mathrm{~V}, 6$, p. $154:$ le personnage reçoit une lettre d'Illus l'informant de ses projets, et cela par l'entremise de Jean Talaia.

39. Citons DuCHESNE 1910 : « Talaia, soutenu par Illus et Theognoste, fit à Constantinople l'effet d'un intrigant, plus occupé de ses propres affaires que de celles de son église ».

40. Zacharias Rhetor, $H E \mathrm{~V}, 7$, p. 155 : Jean achète son poste à Theognostus, en lui remettant le trésor octroyé jadis par l'empereur Arcadius à l'archevêque Théophile d'Alexandrie: voir DHGE XXVII, «Jean le Tabennèsiote», col. 679-681 (D. STIERNON).

41. Maraval 1998, p. 119-120: Jean Talaia fut consacré par les chalcédoniens ; Calandion d'Antioche avait ignoré l'édit. 
déposition du patriarche monophysite d'Antioche, Pierre le Foulon, qui fut, après bien des péripéties, justement remplacé par Calandion ${ }^{42}$.

\section{Les fidèles de Zénon}

Si la révolte de Leontius fut prioritairement centrée sur l'Isaurie, l'empereur Zénon lui opposa pour sa part un solide réseau de fidélités également isauriennes ${ }^{43}$. C'est d'abord le cas du demi-frère bâtard d'Illus lui-même, Lilingis ${ }^{44}$, qui demeura dans le camp de Zénon. L'empereur lui confia en 484 le commandement de son armée contre la révolte et il est attesté en 491 comme gouverneur de la province d'Isaurie. L'isaurien Indacus Cottunes ${ }^{45}$ endossa, lui, le rôle du traître puisqu'il collabora à la révolte en 484 avant de passer dans le camp impérial en $488^{46}$. Le profil de Conon ${ }^{47}$ est également digne d'intérêt, car le personnage fit une double carrière au service de Zénon : il la débuta et la finit comme militaire, en se voyant également confier les troupes impériales en 484 ; mais, entre-temps, il détint le siège épiscopal d'Apamée, où il est encore attesté en 484 et où il recueillit peutêtre des informations sur la révolte. Enfin, Longinus ${ }^{48}$, né à Cardala en Isaurie, fut nommé maître des offices en 484 : c'était visiblement l'un des protecteurs de ses compatriotes présents à Constantinople ${ }^{49}$.

42. Downey 1961, p. 489-490 et Gray 1979, p. 27.

43. Zénon avait déjà particulièrement prodigué ses bienfaits à l'Isaurie à la suite de la révolte de Basiliscus : d'après Evagrius III, 8, Zénon se serait vu promettre son rétablissement par une prédiction recueillie dans le monastère isaurien de SainteThècle, et l'aurait doté d'une nouvelle église en remerciement. Selon DAGRON 1978, p. 59, une « séduisante hypothèse » consisterait à placer cette construction dans une série d'autres fondations de Zénon dans la région, spécialement en Cilicie ; les sites d'Alahan, Dag Pazari, Meryemlik et Korykos ont été étudiés par Gough 1972. En tout cas, à Zenonopolis, en Isaurie, on sait par une inscription que le sanctuaire de Saint-Socrate a bien été enrichi par Zénon en 488, donc l'année où se termine la révolte de Leontius : MARAVAL 1985, p. 358.

44. PLRE : LILINGIS.

45. PLRE : Indacus Cottunes. Le nom Indacus est répandu en Isaurie : il est porté par un évêque de Korykos en 515/518 (Sévère d'Antioche, V, 7).

46. Malalas, fragments : 214 .

47. PLRE : Conon 4. Le nom même de Conon renvoie à un saint local; voir HaLKIN 1985 : il y aurait eu deux saints isauriens de ce nom, dont l'un au moins fut martyrisé sous Aurélien.

48. PLRE : Longinus 3.

49. Théophane, p. 21. Et en effet, selon SHaw 1990, p. 254, «In this jockeying for power, the Isaurians in the capital continued to exploit friends and relatives as a principal resource ». 


\section{Le règne d'Anastase (491-518)}

\section{L’opposition des anciens fidèles de Zénon}

La rébellion isaurienne se poursuivit sous le règne d'Anastase. Or il est frappant de constater que ce sont précisément les fidèles isauriens de Zénon qui en devinrent les chefs sous son successeur : on dispose ainsi de la preuve que ces personnages jouaient de leur identité isaurienne en fonction des changements politiques et qu'elle n'était donc pas une essence intemporelle ${ }^{50}$. Ainsi, Lilingis, Conon et Longinus furent tous trois défaits par l'armée d'Anastase à la bataille de Cotyaeum en 492. Le caractère précoce de cette révolte au cours du règne d'Anastase laisse bien penser que ses chefs étaient mus par une forme de fidélité à l'empereur défunt. Il est patent que la rébellion isaurienne vint en effet appuyer les prétentions impériales du propre frère de Zénon, Longinus ${ }^{51}$ : le personnage fut emprisonné en 492 et banni en Égypte. En outre, cette révolte reçut manifestement l'appui du patriarche Euphemius ${ }^{52}$ et fut donc peut-être pro-chalcédonienne ${ }^{53}$.

\section{Les fonctionnaires monophysites d'Anastase}

On sait que, tout en restant fidèle officiellement à l'Hénotique édicté par Zénon, son successeur Anastase tendit à accorder sa faveur à des ecclésiastiques monophysites ${ }^{54}$. De la même manière, il est notable que les monophysites furent largement représentés au sein de l'administration civile d'Anastase. S'y dessine nettement en premier lieu un groupe de personnages liés au grand théologien monophysite Sévère, devenu évêque d'Antioche à la fin du règne, en 512. Ainsi,

50. Burgess 1992 souligne que les Isauriens Zénon et Illus se sont parfaitement insérés dans une compétition traditionnellement romaine pour le pouvoir. La démonstration gagne en force à être étendue au règne d'Anastase. Mais il nous semble que l'auteur tombe parfois dans le travers qu'il dénonce, une vision ethnique des Isauriens : beaucoup de personnages cités par lui comme Isauriens le sont de manière incertaine, par un raisonnement circulaire basé sur leur proximité avec Zénon ou Illus !

51. PLRE : Fl. Longinus 6. Evagrius III, 29, dit d'abord que Longinus et beaucoup d'autres Isauriens furent expulsés de la capitale par Anastase à la suite du complot. Le même Evagrius III, 35, affirme plus loin que l'autre Longin [de Cardala] était bien la « force principale de la faction usurpatrice » (trad. A.-J. Festugière).

52. Théophane, p. 215 : AM 5987=487, donc avant l'avènement d'Anastase.

53. JARRY 1968, p. 289. On a là un exemple des approximations de ce livre : la révolte de 492 est qualifiée de " pro-chalcédonienne » d'après un fait remontant à 487 et selon un raisonnement transférant l'option religieuse d'un personnage à celui qu'il soutenait alors.

54. Maraval 1998, p. 123. 
Anastasius ${ }^{55}$, comte en 513/518, était déjà le fils d'un monophysite, Sergius ${ }^{56}$; luimême faisait partie des correspondants de Sévère d'Antioche ${ }^{57}$. Le consul de 513 Clementinus ${ }^{58}$, comte des largesses sacrées, fut un protecteur constantinopolitain du moine Sévère, futur évêque d'Antioche ${ }^{59}$; en 511 , il prit une part active à la déposition du patriarche chalcédonien de Constantinople, Macedonius ${ }^{60}$. Eupraxius ${ }^{61}$, cubicularius vers 508/511, comptait parmi les partisans de Sévère d'Antioche dans la capitale ${ }^{62}$. Le consul de 512, Paulus ${ }^{63}$, reçut pour sa foi les louanges de Sévère d'Antioche ${ }^{64}$. Enfin, c'est le consul de 502, Probus ${ }^{65}$, neveu d'Anastase, qui introduisit le fameux moine Sévère auprès de l'empereur ${ }^{66}$.

Mais les relations directes de Sévèren'épuisent pas le groupe des fonctionnaires monophysites d'Anastase. Ainsi, Constantinus ${ }^{67}$, monophysite patenté, est attesté

55. PLRE : ANASTASIUS 8.

56. PLRE : Sergius 2.

57. Sévère d'Antioche, III, 1, p. 16 : Anastasius reçoit de Sévère la communion en même temps que des éloges sur la ferveur et la pureté de foi de son père. Anastasius était peut-être ce pieux monophysite à qui Sévère demanda à des correspondants à Antioche de donner la communion.

58. PLRE : Fl. Taurus Clementinus Armonius Clementinus.

59. Zacharias Rhetor, Vita Severi, 27, p. 104.

60. Zacharias Rhetor, $H E$ VII, 8.

61. PLRE : EUPRAXIUS.

62. Zacharias Rhetor, Vita Severi, 27, p. 104 : cité par la source en même temps que le précédent personnage. Eupraxius discuta de problèmes dogmatiques avec Sévère, qui lui dédia des traités de théologie : Idem, 27, p. 106.

63. PLRE : Fl. Paulus 34.

64. Sévère d'Antioche, Epistulae, 118, p. 291 : Paulus est du côté de Sévère dans un débat sur l'acceptation du concile de Chalcédoine par des monastères.

65. PLRE : Fl. Probus 8. Sur le lien de parenté entre Anastase et Probus : voir en dernier lieu SetTiPani 2000, p. 420.

66. Zacharias Rhetor, $H E$ VII, 10 : la source laisse comprendre que c'est donc grâce à Probus que Sévère devint évêque d'Antioche.

67. PLRE : Constantinus 15. 
comme maître des milices avant 510. Marinus ${ }^{68}$, préfet du prétoire d'Orient, vit sa maison de Constantinople incendiée par la foule chalcédonienne en $512^{69}$.

\section{Les fonctionnaires syriens d'Anastase}

Il est notoire que les principaux inspirateurs ecclésiastiques d'Anastase à la fin de son règne étaient des monophysites syriens ${ }^{70}$. Aussi est-il intéressant de se demander dans quelle mesure une telle orientation put s'insérer dans une faveur plus large accordée à des élites de cette région de l'Empire. Et en effet le groupe le plus fourni au sein des fonctionnaires orientaux d'Anastase était originaire d'Antioche. On a d'abord affaire à la famille de Calliopius ${ }^{71}$ qui est vicarius du maître des milices d'Orient en 513/518. Le rôle de ce personnage au service d'Anastase fut tout à fait direct puisqu'il est connu pour avoir systématiquement soutenu les monophysites ${ }^{72}$. Son fils Theodosius ${ }^{73}$ se retrouve préfet augustal à Alexandrie : en 516, il est tellement identifié au pouvoir impérial que lorsque le patriarche Dioscore est élu avec le soutien d'Anastase, Theodosius est massacré par la foule. Enfin, cet antiochéen Calliopius était l'oncle de l'évêque de Beroia, Antoninus ${ }^{74}$, attesté en 513/518 comme correspondant de Sévère d'Antioche ${ }^{75}$ : ce personnage détenait évidemment un siège épiscopal très proche du berceau familial. Parmi les Antiochéens, on trouve encore deux comites Orientis de 507 : le premier, Irenaeus ${ }^{76}$, acquit une réputation de terreur en s'attaquant aux menées des factions dans sa cité natale ; le second, Procopius ${ }^{77}$, fut moins chanceux car il du abandonner son quartier général sous la pression de la foule.

\section{PLRE : Marinus 7.}

69. En outre, l'un des parents de Marinus fut recommandé pour la prêtrise par Sévère d'Antioche à l'évêque d'Apamée Étienne : Sévère d'Antioche, VII, 6. On se trouve donc bien avec la parentèle de Marinus en milieu monophysite.

70. Maraval 1998, p. 126.

71. PLRE : Calliopius 6 : né à Antioche, bâtisseur de la forteresse de Dara en 505/506.

72. Sévère d'Antioche, I, 45.

73. PLRE : THEODOSIUS 18 : né à Antioche.

74. PLRE : Antoninus 1.

75. De même que Sévère, Antoninus fut exilé à Alexandrie lors du retour au chalcédonisme de 518.

76. PLRE $:$ IRENAEUS $5=$ PENTADIASTES : né à Antioche.

77. PLRE : PROCOPIUS 6 : né à Antioche. 
Un groupe de taille équivalente d'après nos sources provenait de villes très proches d'Antioche. Ainsi, Constantius ${ }^{78}$, comes Orientis : né à Tarse, c'est à Antioche qu'il reçut des pouvoirs extraordinaires après la révolte de la faction verte en 494. Marinus ${ }^{79}$, né à Apamée, reçut le poste de préfet du prétoire d'Orient, qui le vit en particulier commander en 515 les troupes impériales envoyées contre le rebelle Vitalien. Quant à Polycarpus ${ }^{80}$, également préfet du prétoire en 498, il était né à Berytus, mais entretenait d'étroites relations avec Sévère d'Antioche : probablement à la fin des années 480 , il s'attaqua à des pratiques magiques en compagnie du futur prélat ${ }^{81}$. Enfin, Sergius ${ }^{82}$, un autre préfet du prétoire en 517, était originaire de Zeugma. On peut donc conclure qu'une cohérence notable s'est manifestée entre les options religieuses d'Anastase et la région d'origine de nombre de ses collaborateurs : sans qu'il s'agisse d'accorder à l'un de ces deux aspects une valeur explicative de l'autre, leur concomitance révèle bien la cohésion d'un réseau aristocratique ${ }^{83}$.

\section{Les fidèles chalcédoniens d'Anastase}

Malgré la faveur accordée aux monophysites, la ligne religieuse officielle d'Anastase excluait une chasse systématique aux ecclésiastiques chalcédoniens. C'est ainsi que le patriarcat de Constantinople lui-même fut occupé par deux partisans convaincus du concile de 451, Euphemius et Macedonius ${ }^{84}$. De même, les opinions chalcédoniennes de membres de la famille d'Anastase furent tolérées par l'empereur ${ }^{85}$. Il semble même qu'il se soit agi en l'occurrence pour

78. PLRE : CONSTANTIUS $13:$ né à Tarse.

79. PLRE : Marinus 7 : né à Apamée.

80. PLRE : Polycarpus : né à Berytus.

81. Zacharias Rhetor, Vita Severi, 15, p. 59-60 et 17, p.65.

82. PLRE : Sergius 7 : né à Zeugma.

83. On peut remarquer que cette cohésion géographique est sans rapport avec la région d'origine d'Anastase, en l'occurrence l'Épire, où le futur empereur fut pourtant un propagandiste du monophysisme (GRILLMEIER 1990, p. 372). La carrière d'Anastase avant son avènement présente beaucoup d'incertitudes, mais il est assuré qu'il fut vers 488 un candidat possible à la succession de Pierre le Foulon au patriarcat d'Antioche: des liens avec les monophysites syriens se manifestaient donc bien déjà.

84. Maraval 1998, p. 124-125.

85. CHARANIS 1974,p. 18, signalait déjà que plusieurs personnalités défendaient le concile de Chalcédoine à l'avènement d'Anastase : Iuliana fille d'Olybrius, Placidia petitefille de Theodose II, Pompeius neveu d'Anastase et sa femme, et enfin l'épouse du frère d'Anastase. 
le pouvoir de préserver l'appui d'un réseau de parents prestigieux ${ }^{86}$. Était ainsi chalcédonienne Magna ${ }^{87}$, épouse d'un frère de l'empereur ${ }^{88}$. De même, le neveu de l'empereur Hypatius ${ }^{89}$, l'un des maîtres des milices les plus en vue du règne, était un chalcédonien notoire, refusant la communion de Sévère d'Antioche ${ }^{90}$. Son frère, un autre neveu d'Anastase donc, Pompeius ${ }^{91}$, consul en 501, non seulement fréquentait les moines chalcédoniens ${ }^{92}$, mais il donna assistance au patriarche exilé Macedonius ${ }^{93}$. Sa propre épouse, Anastasia ${ }^{94}$, faisait également partie des intimes de Sabas lors de son séjour dans la capitale en 511/512. Lors de ses visites au saint homme, elle était accompagnée de Iuliana ${ }^{95}$. Or cette dernière, chalcédonienne dévote comme Anastasia, reçut des pressions infructueuses de la part de l'empereur et du patriarche Timothée dans le but de modifier sa foi ${ }^{96}$. Il faut dire que son cas est quelque peu particulier puisque c'est dans sa demeure constantinopolitaine que la foule chalcédonienne a tenté de porter au pouvoir en 512 son propre époux Areobindus ${ }^{97}$, maître des milices d'Orient en 503-504 et

86. Мс Совміск 2000, p. 148, remarque plus largement que neuf parents d'Anastase obtinrent le consulat entre 500 et 518 .

87. PLRE : Magna.

88. Selon CAmERon 1978, ce frère est bien Paulus 26, alors que les éditeurs de la PLRE hésitaient à rapprocher les deux personnages. Magna reçut du moine chalcédonien d'Alexandrie Dorothée un traité de défense du concile de 451 qu'elle présenta à Anastase, dans l'espoir, selon le chroniqueur Théophane, de changer ses vues : Théophane, p. 235 (AM 6002=502).

\section{PLRE : Fl. Hypatius 6.}

90. Cyrille de Scythopolis, p. 152 : Hypatius assure qu'il n'est pas en communion avec Sévère, mais avec le patriarche de Jérusalem Elias et les moines Théodose et Sabas, au moment où ces derniers anathématisent dans la Ville Sainte, en 516, ceux qui n'acceptent pas le concile de Chalcédoine.

91. PLRE : Pompeius 2.

92. Cyrille de Scythopolis, p. 145 : Pompeius se trouve sur le Mont des Oliviers à Jérusalem pour une retraite monastique, effectuée donc en milieu chalcédonien.

93. Théophane, p. 243 (AM 6005=505).

94. PLRE : Anastasia 3.

95. PLRE : Anicia Iuliana 3. Cyrille de Scythopolis, p. 145: les deux dames se réjouissent de l'enseignement divin de Sabas.

96. Selon Théophane p. 243 (AM 6005=505), il s'agit précisément de l'amener à communier avec le patriarche.

97. PLRE : Fl. Areobindus Dagailaphus Areobindus 1. 
consul en 506. Il est néanmoins significatif que ce personnage ait refusé de rompre la fidélité à l'empereur, manifestant ainsi la solidité de ce réseau chalcédonien au service d'Anastase. Un dernier personnage témoigne de l'utilisation des chalcédoniens par Anastase. Il s'agit de Celer ${ }^{98}$, maître des offices en 503-518, qui se mit en toutes circonstances au service de la politique religieuse de l'empereur. D'un côté, en effet, il participa à la déposition du patriarche Macedonius : il réussit à lui faire signer un document ignorant les deux conciles d'Éphèse et Chalcédoine, lui aliénant ainsi à la fois le monophysisme impérial et le chalcédonisme du peuple de Constantinople ${ }^{99}$ ! D'un autre côté, Celer obtint de Sévère d'Antioche la réinstallation des évêques chalcédoniens de Syrie Seconde.

\section{La révolte chalcédonienne et européenne de Vitalien}

Le chalcédonisme du fameux rebelle Vitalien ${ }^{100}$ eut évidemment partie liée avec des questions politiques. D'un point de vue privé, le personnage était peutêtre le filleul de Flavien d'Antioche ${ }^{101}$, évêque démis au profit du monophysite Sévère. Les facteurs de la révolte de Vitalien sont certainement multiples, mais on peut au moins en identifier deux. Un élément régional semble avoir tenu la première place : la révolte a été déclenchée en 513 à la suite du refus impérial d'accorder des subsides aux foederati du diocèse de Thrace. Le rebelle reçut alors le soutien des troupes régulières des provinces de Thrace et de Scythie, ainsi que celui de Maxentius duc de Mésie seconde, la province de naissance de Vitalien. En outre, la paysannerie de la région s'est visiblement engagée en masse dans l'armée du rebelle : elle était déjà à coup sûr au moins en partie solidaire des foederati et des soldats. Le second facteur de cette révolte est religieux : le rebelle soutenait le chalcédonisme et, réciproquement, reçut l'appui des chalcédoniens dans les zones contrôlées ${ }^{102}$. Or le but de Vitalien était manifestement de défendre ces deux causes auprès d'Anastase ${ }^{103}$. Il accepta en effet en 514 de cesser sa rébellion,

\section{PLRE : Celer 2.}

99. Celer eut en outre la lourde et infructueuse tâche de tenter de pacifier la foule chalcédonienne de la capitale en 512 : Marcellinus Comes 512.

\section{PLRE : Fl. Vitalianus 2.}

101. Zacharias Rhetor., HE, VIII, 2, p. 43.

102. Théophane, p. 242, affirme même en présentant la révolte que ce sont les orthodoxes de Scythie, Mésie et des autres régions qui appelèrent Vitalien à se soulever contre «l'impie Anastase ».

103. Charanis 1974 , p. 51, a bien noté qu'un lien entre les deux facteurs préexistait à la révolte elle-même: les régions danubiennes étaient mécontentes des options monophysites d'Anastase. GRAY 1979, p. 41, va plus loin : « Scythia, Illyricum, and Lower Moesia had had a continuous and strong tradition of pro-Chalcedonianism. Scythian monasteries, for instance, remained in communion with Rome throughout 
en échange de l'obtention du poste de maître des milices de Thrace, ainsi que du rappel des évêques chalcédoniens exilés, Flavien d'Antioche et Macedonius de Constantinople. Par contre, le refus d'Anastase de réunir un concile associant le Pape conduisit à une rupture en 515 entre l'empereur et le rebelle. Mais on constate ainsi que le chalcédonisme de Vitalien constitua moins une option radicalement antagoniste de la politique impériale qu'un aspect d'une négociation avec le pouvoir. Du point de vue de l'empereur, il est significatif que le principal officier chargé de s'opposer à cette révolte ait été justement un chalcédonien, le neveu d'Anastase déjà évoqué, Hypatius. Il est possible que l'empereur ait ainsi trouvé un moyen de se concilier le rebelle, car c'est bien au moment de la capture d'Hypatius par Vitalien, et en échange de sa libération contre rançon, que s'est instauré le compromis de 514.

\section{La continuité des familles de fonctionnaires des deux règnes}

En dépit des ruptures évoquées, la transition entre les règnes de Zénon et Anastase se caractérisa aussi par une continuité certaine, sur le plan de la théologie officielle, mais également en ce qui concerne le recrutement du personnel politique ${ }^{104}$. On peut en effet relever une série de lignages dont les représentants traversèrent les deux règnes au sommet du pouvoir. En fait, il est frappant de noter que dans bien des cas, ils héritèrent leur position d'une génération encore antérieure, à l'époque de Léon $1^{\mathrm{er}}$. Cet élément tend à montrer que si le règne de l'époux de Vérine fonda des contestations ultérieures du pouvoir impérial, il installa aussi certaines familles de manière durable au sein de l'aristocratie. Deux exemples illustrent cette continuité lignagère. Ainsi, Vivianus ${ }^{105}$, préfet du prétoire en 459-460 (sous Léon $1^{\mathrm{er}}$ ), était le père d'Adamantius ${ }^{106}$, préfet de la Ville de Constantinople en 474/479 (sous Zénon), et de Paulus, consul en 512 (sous Anastase). D'autre part, Dagailaphus ${ }^{107}$, consul en 461 (sous Léon $1^{\mathrm{er}}$ ), avait pour fils Areobindus Dagailaphus, maître des milices en 503-504 (sous Anastase), qui lui-même était le père de Olybrius, consul en 491.

the Acacian schism. It was on this tradition in his native provinces that Vitalian based his revolt ».

104. La première continuité fut évidemment assurée par l'impératrice Ariane, épouse successive de Zénon et Anastase : Evagrius dit plaisamment qu'Anastase « épousa à la fois le pouvoir de Zénon et Ariane » (trad. A. J. Festugière).

105. PLRE : Fl. Vivianus 2.

106. PLRE : Adamantius 2.

107. PLRE : Fl. Dagailaphus 2. 


\section{Conclusion}

Le visage des élites sous les règnes de Zénon et Anastase peut être récapitulé à travers le prisme urbain. La capitale est d'abord le lieu où se modèle la dynastie impériale : cette logique constantinopolitaine fonde les revendications mais aussi les compromis au sein de la parentèle de l'empereur. Dans les villes des provinces se noue un lien entre des élites locales et des options religieuses, souvent fortement enracinées dans des particularismes. Mais ces liens organiques entre des villes et des élites ne sont pas seulement statiques. Ils engendrent une dynamique des relations politiques au sein de l'Empire romain d'Orient. Le pouvoir de la capitale modèle ainsi la physionomie des élites urbaines provinciales, en particulier par la nomination des fonctionnaires voire des évêques. Mais réciproquement les villes de l'Empire, associées à leurs territoires civiques et provinciaux, pèsent sur le recrutement du Sénat et de la Grande Église. Les protagonistes d'une révolte ou d'une usurpation sur des bases locales peuvent devenir les maîtres de la capitale. Ainsi, l'Empire romain d'Orient fut bien le créateur d'une élite impériale assurant unité, cohésion et même centralisation au profit de Constantinople. Mais dans le même temps, cet État n'a pu, ni su ni peut-être voulu briser la pluralité de ses élites urbaines provinciales. Il semble même au contraire que la vitalité et la diversité des élites locales comptèrent parmi les conditions de la pérennité d'une capitale.

Vincent PueCH

Université de Versailles-Saint-Quentin UMR 8167

\section{Références bibliographiques}

Blaudeau 1996

$\mathrm{Ph}$. Blaudeau, «Timothée Aelure et la direction ecclésiale de l'empire postchalcédonien », Revue des Études Byzantines 54 (1996), p. 107-133.

BuRgESS 1992

W.D. Burgess, «Isaurian Factions in the Reign of Zeno the Isaurian », Latomus 51 (1992), p. 874-880.

CAMERON 1978

Al. Cameron, « The House of Anastasius », Greek, Roman and Byzantine Studies 19 (1978), p. 259-276.

Charanis 1974

P. Charanis, Church and State in the later Roman Empire: The Religious Policy of Anastasius I, réédition, Thessalonique (1974).

Cyrille de Scythopolis

Cyrille de Scythopolis, Vita Sabae, éd. E. Schwartz, dans Texte und Untersuchungen (1939). 
DAGRON 1978

G. DAgron, Vie et miracles de sainte Thècle, Bruxelles (1978).

DAGRON 1996

G. Dagron, Empereur et prêtre. Étude sur le «césaropapisme » byzantin, Paris (1996).

\section{Demandt 1989}

A. Demandt, Die Spätantike : römische Geschichte von Diocletian bis Justinian, Münich (1989).

DHGE

Dictionnaire d'Histoire et de Géographie Ecclésiastique.

Downey 1961

G. Downey, A History of Antioch in Syria from Seleucus to the Arab Conquest, Princeton (1961).

DuCHESNE 1910

L. DuCHESne, Histoire ancienne de l'Église, Tome III, Paris (1910).

ELTON 2000

H. Elton, «Illus and the Imperial Aristocracy under Zeno », Byzantion 70 (2000), p. 393-407.

Evagrius

Evagrius, Historia Ecclesiastica, éd. J. Bidez et L. PARMEnTIER (1898).

FeISSEL 1999

D. FeISSEL, « Deux grandes familles isauriennes du v viècle d'après des inscriptions de Cilicie Trachée », Mitteilungen zur Christlichen Archäologie 5 (1999), p. 9-17.

Gouch 1972

M. Gouch, «The Emperor Zenon and some Cilician Churches », Anatolian Studies 22 (1972), p. 199-212.

Gray 1979

P.T.R. Gray, The Defense of Chalcedon in the East (451-553), Leyde (1979).

GRILLMEIER 1990

A. GrillmeIer, Le Christ dans la tradition chrétienne. Tome II/1. Le concile de Chalcédoine (451) : réception et opposition, trad. fr., Paris (1990).

HALKIN 1985

F. Halkin, « Vie de S. Conon d'Isaurie », Analecta Bollandiana 103 (1985), p. 5-34.

JARRY 1968

J. JARRY, Hérésies et factions dans l'Empire byzantin du IV au VII siècle, Le Caire (1968).

LEMERLE 1963

P. Lemerle, «Fl. Appalius Illus Trocundes », Syria 40 (1963), p. 315-322.

LESZKa 2000

M.J. LeszKA, « Armatus : a story of a Byzantine general from the $5^{\text {th }}$ century », Eos 87 (2000), p. 335-343. 
Malalas, fragments

Excerpta historica iussu imperatoris Constantini Porphyrogeniti confecta IV, de insidiis, éd. C. DE BoOR (1905).

Maraval 1985

P. Maraval, Lieux saints et pèlerinages d'Orient, Paris (1985).

Maraval 1998

P. Maraval, «La réception de Chalcédoine dans l'empire d'Orient », dans L. Pietri et alii, Histoire du Christianisme, tome III : Les Eglises d'Orient et d'Occident (432610), Paris (1998).

Marcellinus Comes

Marcellinus Comes, Chronicon, éd. Th. Mommsen, dans Monumenta Germaniae Historica (Auctores Antiquissimi), XI (1894).

Mc CoRmick 2000

M. Mc Cormick, «Emperor and court», dans Av. CAMEron et alii, Late Antiquity :

Empire and successors, A. D. 425-600, Cambridge (2000).

PLRE

J.R.Martindale, The Prosopography of the Later Roman Empire, Volume II, A. D. 395-527, Cambridge (1980).

Procope

Procope, La guerre contre les Vandales, trad. D. Roques, Paris (1990).

REDIES 1997

M. Redies, « Die Usurpation des Basiliskos (475-476) im Kontext der aufsteigenden monophysitischen Kirche », Antiquité Tardive 5 (1997), p. 211-221.

SETTIPANI 2000

Ch. SetTIPAni, Continuité gentilice et continuitéfamiliale dans les familles sénatoriales romaines à l'époque impériale. Mythe et réalité, Oxford (2000).

Sévère d'Antioche

Sévère d'Antioche, The Sixth Book of the Select Letters of Severus, patriarch of Antioch, trad. W. Brooks (1902-1904).

Sévère d'Antioche, Epistulae

Sévère d'Antioche, Epistulae, éd. W. Brooks, dans Patrologia Orientalis 14 (1919).

SHAW 1990

B.D. SHAw, «Bandit highlands and lowland peace : the mountains of Isauria-Cilicia », Journal of Social and Economic History of the Orient 33 (1990), p. 237-270.

Théophane

Théophane, Chronographia, Bonn (1839).

TROMBLEY 1993-1994

F.R. Trombley, Hellenic Religion and Christianization c. 370-529, Leiden (19931994).

Zacharias Rhetor, $H E$

Zacharias Rhetor, Historia Ecclesiastica, éd. W. BRooKs, dans Corpus Scriptorum

Christianorum Orientalium (Scriptores Syri), Volumes 87 et 88 (1919-1924). 
Zacharias Rhetor, Vita Severi

Zacharias Rhetor, Vita Severi, éd. M.-A. Krugener, dans Patrologia Orientalis, 2 (1907). 